\title{
Resistencia de Pinus pinaster Ait. al marchitamiento del pino causado por Bursaphelenchus xylophilus (Steiner \& Burher), Nickle
}

Resistance of Pinus pinaster Ait. to pine wilt disease caused by Bursaphelenchus xylophilus (Steiner \& Burher), Nickle

\author{
Menéndez-Gutiérrez, M..$^{*}$
}




\section{Resumen}

La enfermedad del marchitamiento del pino está causada por el nematodo Bursaphelenchus xylophilus, introducido en España en 2008. Este patógeno ha sido el causante de importantes pérdidas económicas y ecológicas en las masas de pino nativas de aquellos países en los que ha sido introducido. Por tanto, el principal objetivo de esta tesis es la determinación de material vegetal de $P$. pinaster con una baja susceptibilidad a $B$. xylophilus.

Los distintos ensayos realizados muestran que las masas de $P$. sylvestris, $P$. pinaster y $P$. radiata en España podrían verse gravemente afectadas por la enfermedad del marchitamiento del pino, en los lugares donde el clima sea favorable para la enfermedad. Además, las procedencias de $P$. pinaster más susceptibles fueron la Noroeste-Litoral, de la zona costera de Galicia, y la procedencia portuguesa Leiria. Sin embargo se han obtenido valores de heredabilidad moderadamente altos para las familias del programa de mejora genética de $P$. pinaster de Galicia. Por tanto, la mejora genética de la resistencia de $P$. pinaster a B. xylophilus puede ser una de las mejores estrategias para luchar contra la enfermedad.

Palabras clave: Susceptibilidad, heredabilidad, mejora genética, procedencias.

\section{Summary}

The pine wilt disease is caused by the nematode Bursaphelenchus xylophilus, introduced in Spain in 2008. This pathogen has been the cause of significant economic and ecological losses in the native pine stands of those countries where it has been introduced. Therefore, the main objective of this thesis is the determination of plant material of $P$. pinaster with a low susceptibility to B. xylophilus.

The different tests carried out show that $P$. sylvestris, $P$. pinaster and $P$. radiata forests in Spain could be severely affected by pine wilt disease, in places where the climate is favorable for the disease. In addition, the most susceptible $P$. pinaster provenances were the "Noroeste-Litoral", from the coastal area of Galicia, and the Portuguese origin Leiria. However, moderately high heritability values have been obtained for the families of the genetic improvement program of $P$. pinaster de Galicia. Therefore, the genetic improvement of $P$. pinaster resistance to $B$. xylophilus may be one of the best strategies to fight the disease.

Keywords: Susceptibility, heritability, genetic improvement, provenances. 


\section{Introducción}

El nematodo de la madera del pino (NMP) Bursaphelenchus xylophilus (Steiner and Buhrer, Nickle), nativo de América del Norte, es el organismo causante de la enfermedad del marchitamiento del pino. Este patógeno ha ocasionado graves pérdidas medioambientales y económicas en Portugal y en países del este asiático; constituyendo actualmente, una de las mayores amenazas para las masas de pino en todo el mundo.

En España, el NMP fue detectado por primera vez en 2008; y en este momento, la enfermedad se limita a dos focos en la provincia de Pontevedra (Galicia) (Abelleira et al., 2011; Xunta, 2016) y un foco recientemente declarado en Lagunilla, Salamanca (Castilla y León) (EPPO, 2018).

Tanto en España como en Portugal, la principal especie hospedante de NMP es Pinus pinaster Ait.; si bien, también B. xylophilus ha sido detectado en árboles de P. radiata D. Don (Zamora et al., 2015) y P. nigra Arnold (Inácio et al., 2015).

Dada la amenaza que representa este patógeno en España, y particularmente en Galicia, se planteó como objetivo general de la tesis doctoral la determinación de

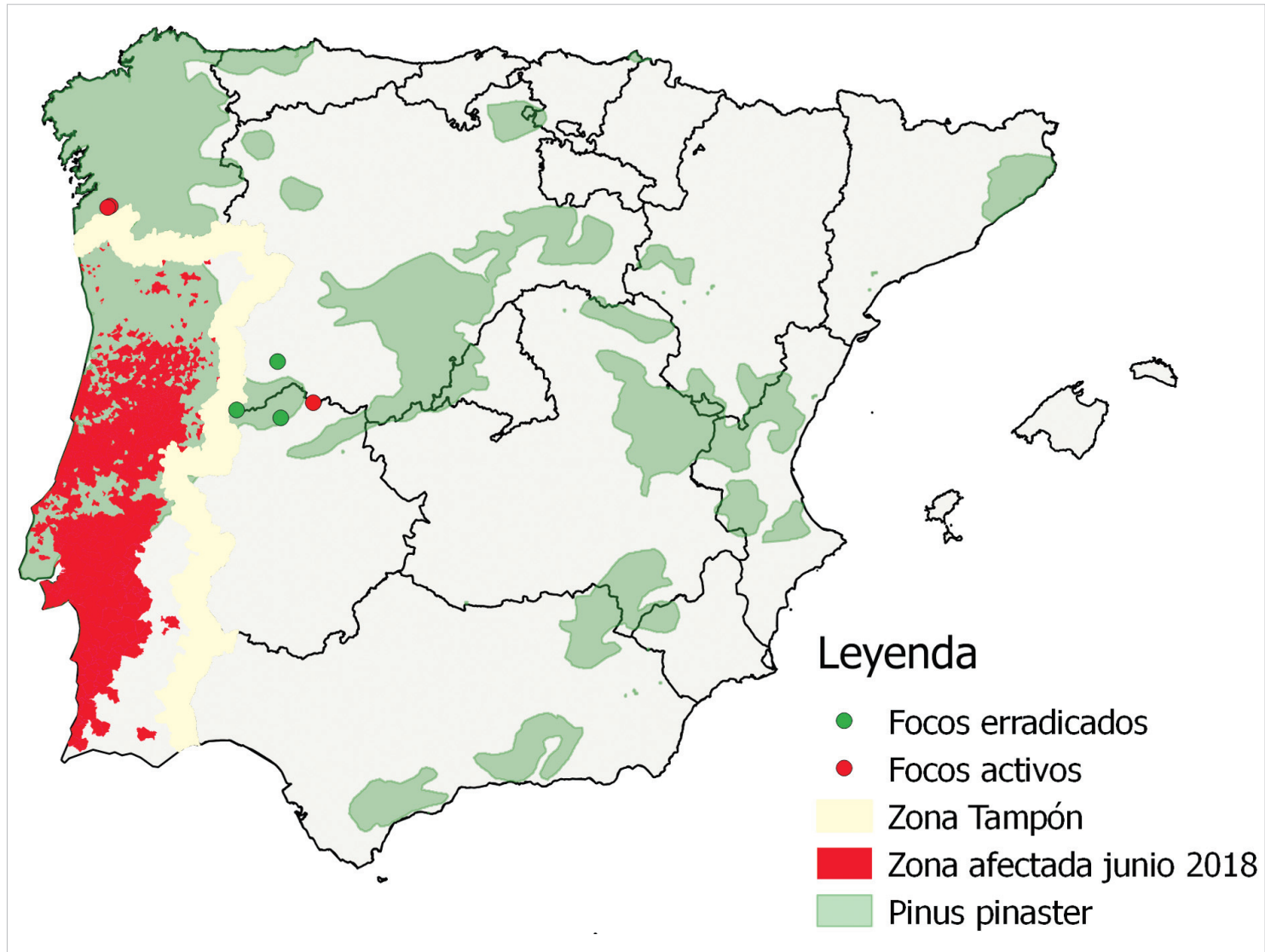

Figura 1. Distribución de la enfermedad del marchitamiento del pino en la península ibérica. En blanco se representa la zona tampón a lo largo de la frontera portuguesa, en rojo las zonas afectadas por $B$. xylophilus en Portugal. El área de distribución de $P$. pinaster en la península ibérica está representado en verde. 
material vegetal de Pinus pinaster tolerante o, al menos, con una baja susceptibilidad a B. xylophilus.

\section{Material y métodos}

Para la determinación del grado en el que las especies de pino podrían verse afectadas en España por NMP, se estudió la variación interespecífica en susceptibilidad al NMP de siete especies de pinos de especial importancia en España: $P$. canariensis Chr. Sm. ex DC., P. halepensis Mill., P. pinaster, P. pinea L., P. sylvestris L., $P$. radiata y $P$. taeda L.; Asimismo, una serie de ensayos fueron realizados para estudiar la variación intraespecifica en diferentes procedencias de $P$. pinaster y familias del programa de mejora genética gallego de esta especie.

Para ello, se inocularon artificialmente con B. xylophilus plántulas de 2 años de edad (Fig. 2) y la evolución de los daños se registró semanalmente siguiendo una escala del 1 al 7 (1, sin daños; 7, muerta) (Fig. 3). Asimismo, se realizaron mediciones de potencial hídrico, fluorimetría, conteo del número de nematodos en raíz y tallo, y se analizaron diferentes compuestos químicos constitutivos en el xilema; todo ello, para cada una de las especies y en diferentes fechas de medición.

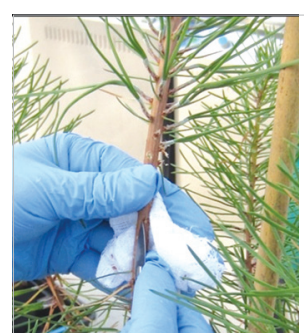

Eliminación acículas

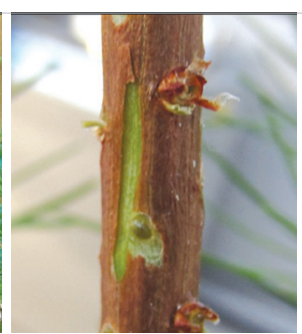

Corte longitudinal

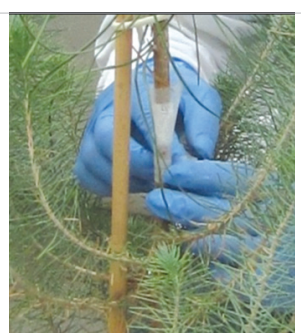

Formación embudo

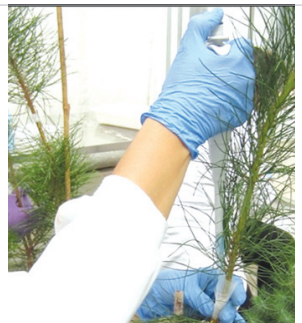

Inoculación

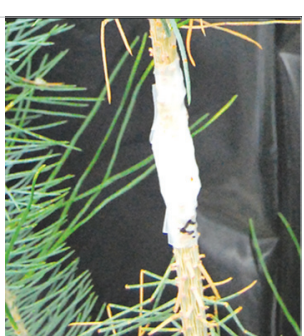

Cierre embudo

Figura 2. Procedimiento de inoculación en plántula.

Fuente: Menéndez-Gutiérrez et al. (2017a).
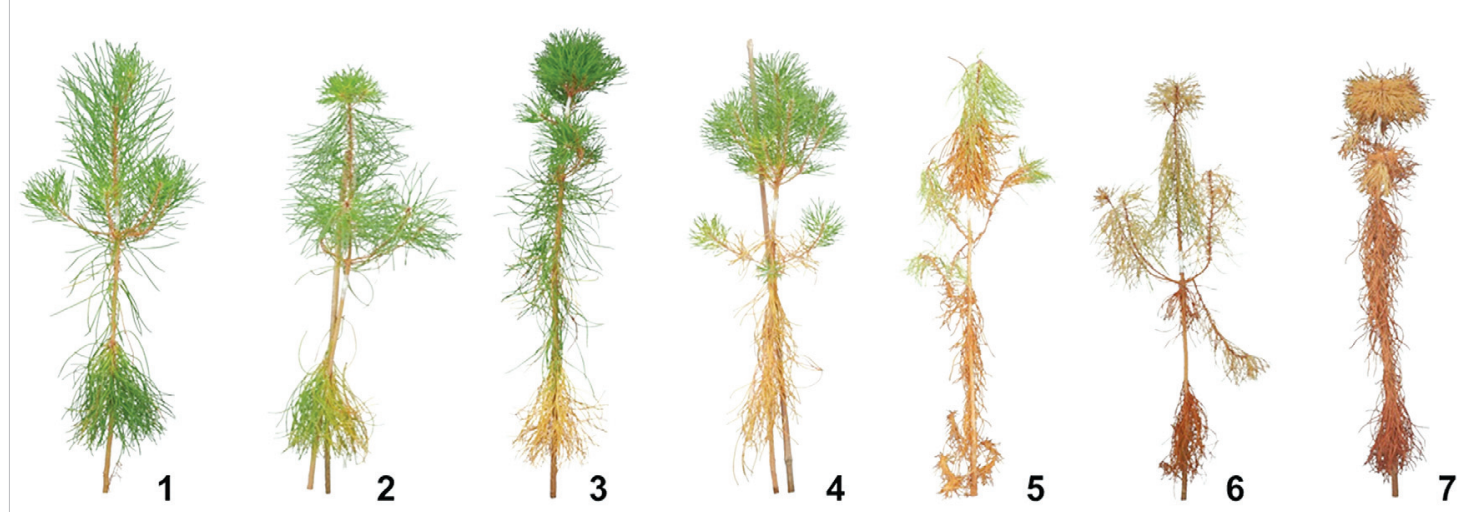

Figura 3. Escala del 1 al 7 para la evaluación periódica de los daños causados por B. xylophilus.

Fuente: Menéndez et al., 2014. 
Además, segmentos de ramas de $P$. taeda y $P$. thunbergii Parl. de diferentes longitudes y clases de tolerancia se inocularon para determinar la relación de estas con la migración.

\section{Resultados y discusión}

Como punto de partida de la tesis, se procedió a evaluar la susceptibilidad de varias especies del género Pinus. A partir de los resultados de mortalidad (Fig. 4) y daños obtenidos, $P$. sylvestris fue clasificada como muy susceptible en consonan-

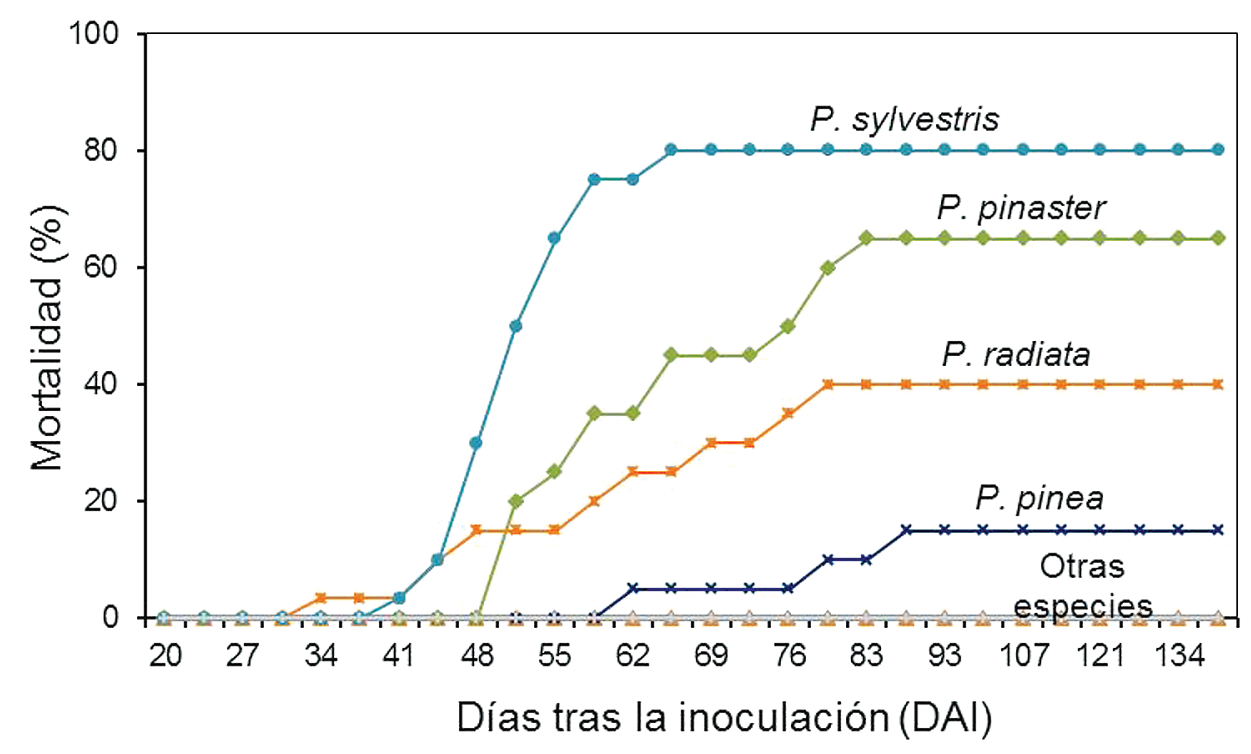

Figura 4. Porcentaje de mortalidad de plántulas de pino tras la inoculación con $B$. xylophilus hasta el final del ensayo. Otras species: P. canariensis, P. halepensis, P. taeda.

Fuente: Menéndez-Gutiérrez et al., 2014.

cia con otras investigaciones, aunque la mortalidad alcanzada no fue tan elevada como la mostrada en otros trabajos (Hopf-Biziks et al., 2016; Panesar and Sutherland, 1989) o como es el caso de las especies asiáticas $P$. thunbergii y $P$. densiflora Sieb. et Zucc. (Mamiya, 1983; Woo et al., 2008). Pinus pinaster y P. radiata se clasificaron como susceptibles, si bien las tasas de mortalidad en algunos estudios difieren ligeramente de las obtenidas en este trabajo (Daub, 2008; Nunes da Silva et al., 2015).

En concordancia con Nunes da Silva et al. (2015), P. pinea se caracterizó por su escasa mortalidad, por lo que fue incluida en el grupo de las especies ligeramente o no susceptibles junto con $P$. canariensis, $P$. pinea, $P$. halepensis y $P$. taeda, las cuales no mostraron mortalidad alguna. A pesar de que otros autores indicaron una mortalidad de hasta un 60\% para P. pinea (Phrame, 2007), en Portugal, esta espe- 
cie está distribuida en zonas afectadas por la enfermedad (Naves et al., 2006) y, por el momento, no se han detectado casos de mortalidad.

En cuanto a $P$. canariensis, es la primera vez que se estudia su susceptibilidad a NMP; y además cabe destacar su condición de ligeramente o no susceptible al ser una especie endémica canaria y de distribución tan restringida.

Seguidamente, dada la presencia de B. xylophilus en Galicia y la gran importancia de $P$. pinaster, tras la clasificación de esta especie como susceptible, se procedió al estudio de la variación en susceptibilidad de procedencias de la península ibérica (cuatro españolas y una portuguesa) y una procedencia francesa.

La fragmentación del área de distribución de $P$. pinaster ha generado una elevada diferenciación entre poblaciones; consecuentemente, se obtuvieron diferencias significativas entre procedencias, en daños por B. xylophilus, mortalidad y los distintos caracteres de historia vital medidos antes de la inoculación. Las procedencias más dañadas, y por tanto con una mayor mortalidad, fueron precisamente las de aquellas zonas en las que NMP está presente, es decir la procedencia portuguesa Leiria y la procedencia de la zona costera de Galicia, Noroeste-Litoral (Fig. 5). En

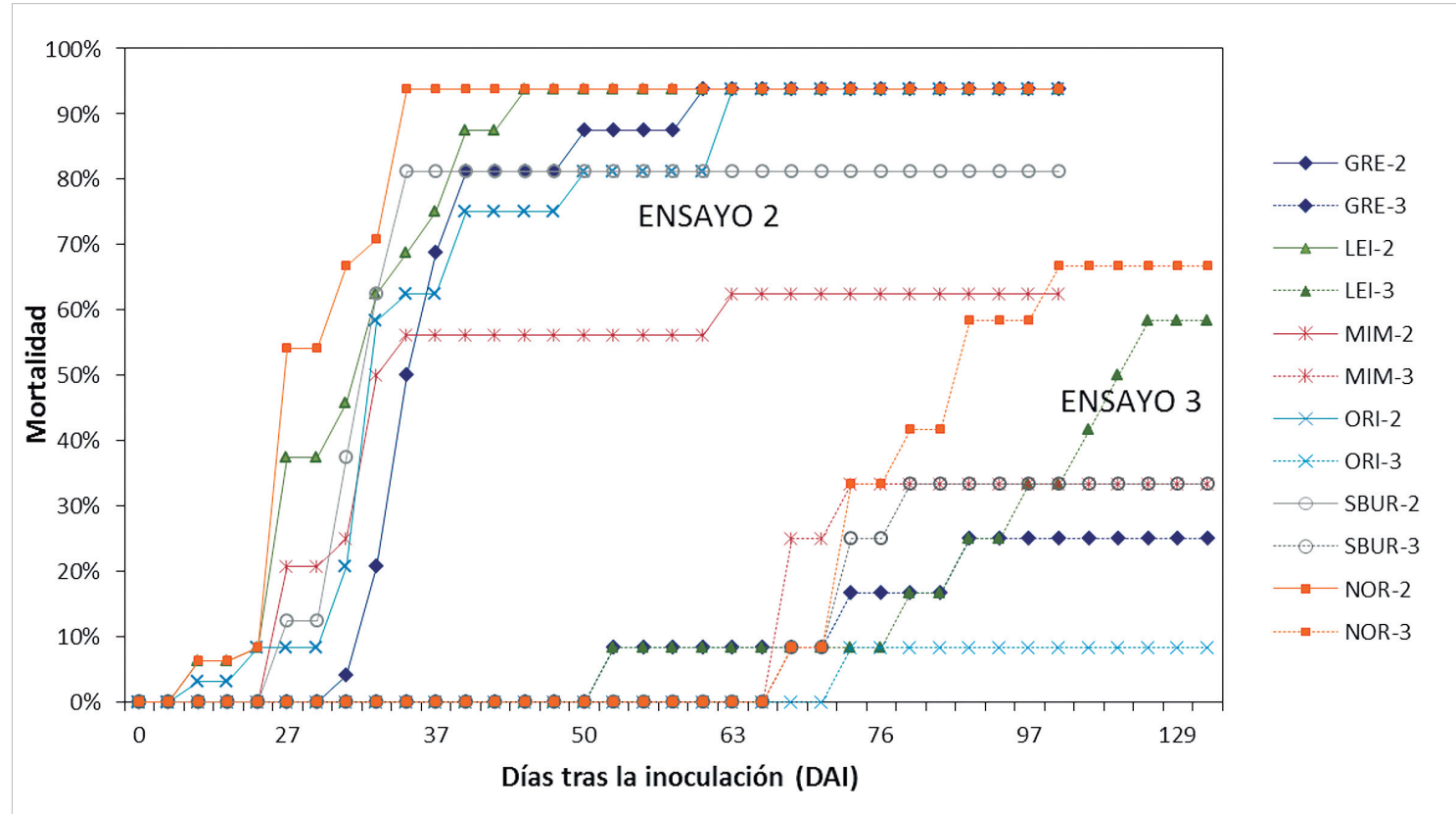

Figura 5. Porcentaje de mortalidad de plántulas de procedencias de P. pinaster tras la inoculación con B. xylophilus hasta el final del ensayo en dos experimentos. GRE: Sierra de Gredos, LEI: Leiria, MIM: Mimizan, ORI: Sierra de Oria, SBUR: Montaña de Soria-Burgos, y NOR: Noroeste-Litoral.

Fuente: Menéndez-Gutiérrez et al. (2017).

relación al resto de procedencias, no ha sido posible determinar con exactitud el nivel de susceptibilidad, debido a la interacción genotipo x ambiente encontrada. Además, se apreció una gran influencia de la temperatura media nocturna y diurna en la expresión de los daños, hipotetizándose también la importancia de la fase de crecimiento vegetativo de la planta en el momento de la inoculación. 
Una vez determinada la moderada susceptibilidad de $P$. pinaster con respecto a otras especies, y la elevada susceptibilidad de la procedencia Noroeste-Litoral, se planteó explorar la variación genética en susceptibilidad a $B$. xylophilus dentro de la población de mejora del programa gallego de mejora genética de $P$. pinaster; y así determinar la posibilidad de incluir la resistencia a la enfermedad como un nuevo carácter de selección con el objetivo de obtener genotipos tolerantes o resistentes a NMP.

En estudios previos en esa misma población de mejora, ya se habían señalado grupos de familias con distinto grado de resistencia al ataque de insectos y hongos (Vivas et al., 2012; Zas et al., 2005). Asimismo, en el caso de la resistencia a $B$. xylophilus, también se han encontrado familias que destacan por su mayor tolerancia. La heredabilidad individual de mortalidad y daños fue moderada $(\sim 0,2)$, no obstante la heredabilidad familiar fue más alta $(\sim 0,6)$, sugiriendo que se pueden esperar importantes ganancias genéticas a través de la selección familiar. Consecuentemente, todo apunta a que la resistencia a $B$. xylophilus puede ser incluida como un nuevo factor de selección en el programa de mejora de $P$. pinaster en Galicia; y así convertirse en una de las estrategias de lucha contra la enfermedad del marchitamiento del pino.

Dentro de un programa de mejora, la determinación de los mecanismos de resistencia a la enfermedad puede ser de ayuda a la hora de seleccionar individuos resistentes o tolerantes. Por tanto, en los distintos ensayos realizados se estudiaron diversos parámetros para ampliar el conocimiento acerca de la enfermedad, así como para tratar de determinar aquellos parámetros que pudiesen estar relacionados con la resistencia: compuestos químicos constitutivos, caracteres de historia vital, variables fisiológicas, así como densidad de nematodos, y desarrollo de la enfermedad.

Los resultados obtenidos establecen cierta correlación entre determinados compuestos químicos constitutivos presentes en el xilema y el nivel de tolerancia a $B$. xylophilus (Tab 1.). Un mayor contenido en nitrógeno y en polifenoles totales pa-

Tabla 1. Matriz de correlación de Spearmana entre los valores de las medias de mínimos cuadrados (LS Means) de mortalidad (M), daños (W), potencial hídrico (WPp: antes de amanecer, WPm: mediodía) y compuestos químicos (POL: polifenoles totales, N: nitrógeno, Mg: magnesio, Fe: hierro, LS: compuestos liposolubles). N=7. Fuente: Menéndez-Gutiérrez et al. (2018a).

\begin{tabular}{|c|c|c|c|c|c|c|c|}
\hline & $\mathbf{M}$ & $\mathbf{P}$ & $\mathbf{M g}$ & $\mathbf{N}$ & $\mathbf{F e}$ & $\mathbf{P O L}$ & $\mathbf{W P m}$ \\
\hline $\mathbf{W}$ & $-0,96^{* * *}$ & $-0,82^{*}$ & & $-0,86^{*}$ & & $-0,82^{*}$ & \\
$\mathbf{M}$ & & $-0,70 \mathrm{a}$ & & $-0,78^{*}$ & & $-0,78^{*}$ & \\
$\mathbf{P}$ & & & $0,71 \mathrm{a}$ & $0,89^{* *}$ & & $0,71 \mathrm{a}$ & \\
$\mathbf{M g}$ & & & & $0,86^{*}$ & $-0,86^{*}$ & & $0,71^{*}$ \\
$\mathbf{N}$ & & & & & & $0,75^{*}$ & $0,71 \mathrm{a}$ \\
\hline $\mathbf{L S}$ & & & & & & & \\
\hline $\mathbf{W P p}$ & & & & & & & $0,96^{* * *}$ \\
\hline
\end{tabular}

Niveles de significación: $* * * \mathrm{p}<0,001 ; * * \mathrm{p}<0,01 ; * \mathrm{p}<0,05$; a $\mathrm{p}<0,10$ 
rece relacionarse con una mayor tolerancia; mientras que altos niveles de compuestos liposolubles y bajos niveles de manganeso con una menor tolerancia a NMP. La mayor parte de los estudios relacionan el mayor contenido de polifenoles y taninos condensados con una mayor resistencia o tolerancia (Nunes da Silva et al., 2015; Pimentel et al., 2016, 2017); sin embargo, un estudio muestra una mayor migración de nematodos en ramas con un mayor contenido en polifenoles totales (Zas et al., 2015).

Otro de los caracteres que parece tener relación con la susceptibilidad es el contenido en resina, la cual constituye la principal defensa de las coníferas frente a agresiones externas, ya sean causadas por patógenos o por factores abióticos (Phillips and Croteau, 1999). En el ensayo de procedencias se observó que aquellas con mayor contenido inicial de resina fueron las menos dañadas. Tanto la mortalidad como los daños se correlacionaron significativamente con el número de ramas y el crecimiento en diámetro, de forma que los genotipos más tolerantes resultaron tener mayor crecimiento en diámetro y mayor ramosidad. Sin embargo, estas relaciones requieren mayor estudio, ya que no fueron encontradas en todos los ensayos en los que se estudiaron; además, si bien existen trabajos que apoyan nuestros resultados (Kuroda, 2004; Toda and Fujimoto, 1987), también hay otros que los contradicen (Hakamata et al., 2013; Yamanobe, 2009).

Con respecto a los parámetros flujo de resina, potencial hídrico y fluorescencia, todos ellos son fieles indicadores del estado fisiológico de las plantas; estableciéndose en la mayoría de los casos, una clara relación con los daños y la mortalidad (Fig. 6). Las mediciones de potencial hídrico realizadas en el ensayo de especies revelaron daños en $P$. sylvestris con anterioridad al inicio del marchitamiento de las acículas; sin embargo, esto no fue observado en el resto de las especies, pro-
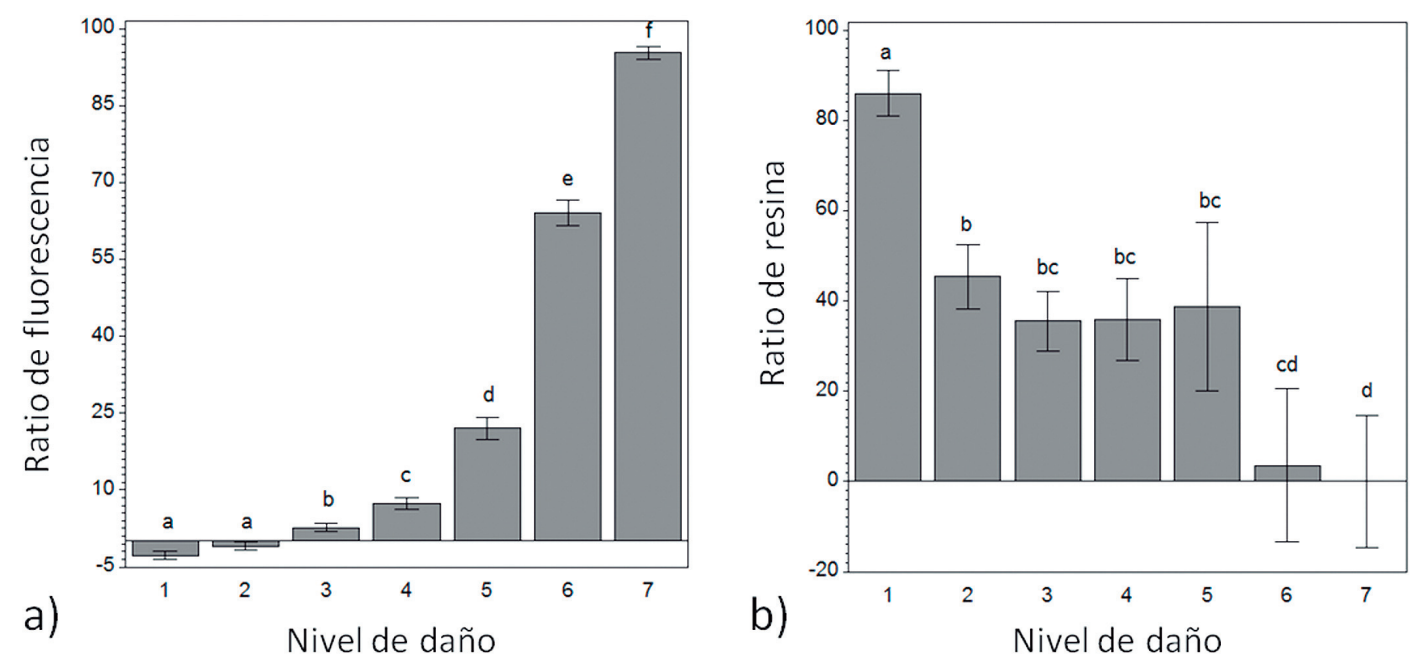

Figura 6. Ratio de fluorescencia (a) y ratio de resina (b) por nivel de daño en plántulas de P. pinaster. LS means \pm SE. Las diferentes letras indican diferencias significativas a $\mathrm{p}<0.05$.

Fuente: Menéndez-Gutiérrez et al. (2018b). 
bablemente por falta de un mayor número de mediciones intermedias. Asimismo, en el ensayo de familias se observó una reducción inicial de producción de resina antes de que ningún síntoma fuese visible y ésta se detuvo completamente antes de que toda la planta se marchitase; la medición del flujo de resina es tradicionalmente uno de los métodos más utilizados en la detección temprana de la enfermedad (Linit and Kinn, 1993; Phrame, 2007).

Otro aspecto a resaltar concierne a la migración y multiplicación de nematodos, factores considerados como relevantes en el desarrollo de la enfermedad por numerosos autores (Mori et al., 2008; Son and Moon, 2013). Nuestros resultados muestran que, si bien la migración ha sido posible en todas las especies estudiadas, se ha registrado siempre con un número menor de nematodos en las especies menos susceptibles. En relación a la multiplicación de nematodos, destacar que en todos los ensayos realizados existió una elevada correlación entre el número de nematodos recuperados y el nivel de daños de las plantas. Además, no puede decirse que haya habido multiplicación en las especies ligeramente o no susceptibles, ya que el número de nematodos recuperados ni siquiera se aproximó al número inoculado (Fig. 7).

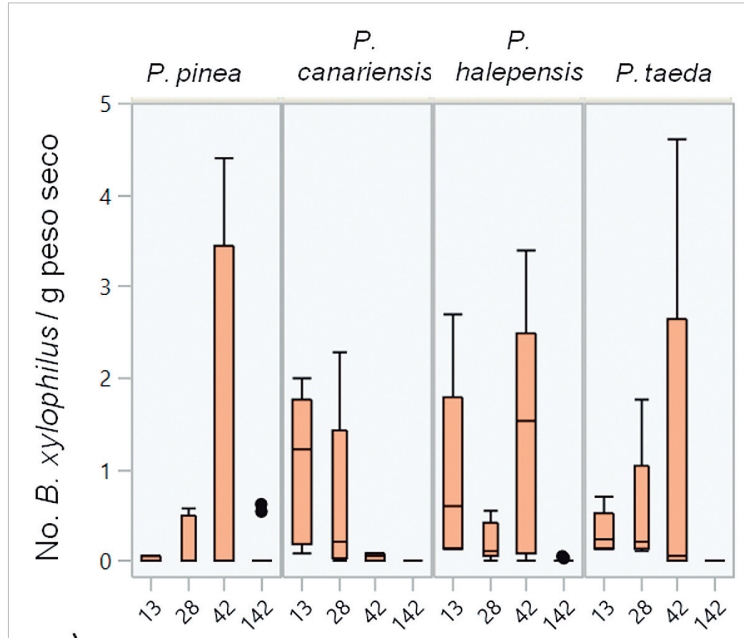

a)

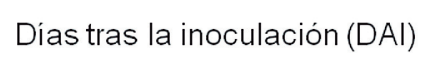

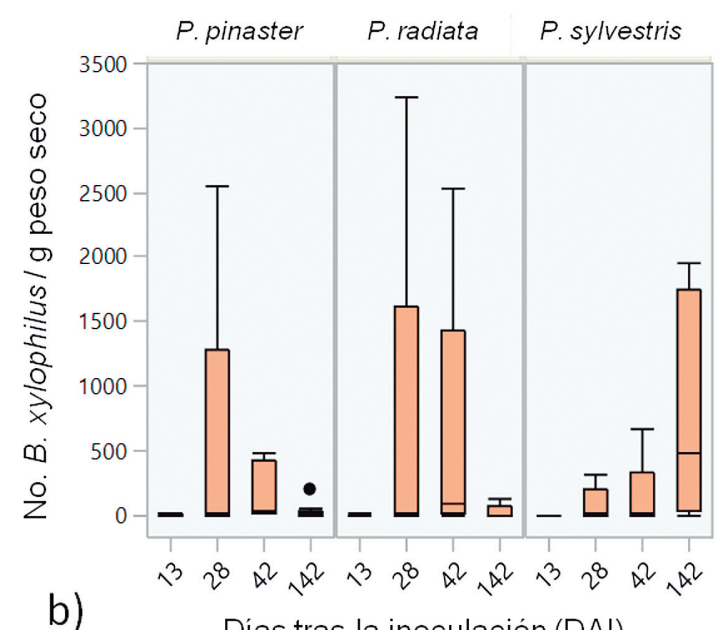

Días tras la inoculación (DAI)

Figura 7. Bursaphelenchus xylophilus por gramo de peso seco. (a) Especies de pino con valores bajos de B. xylophilus por gramo de peso seco. (b) Especies de pino con los valores más altos de B. xylophilus. Diagrama de cajas, Línea (mediana), Caja (25 \% - 75 \% valor de los cuartiles), Bigote (intervalo mínimo-máximo de valores). A: Pinus pinea, C: P. canariensis, H: P. halepensis, P: P. pinaster, R: P. radiata, S: P. sylvestris, T: P. taeda.

Por otro lado, uno de los puntos débiles de los programas de mejora de resistencia a $B$. xylophilus es el tiempo y los medios requeridos para la realización de las consecutivas inoculaciones artificiales necesarias para la selección de individuos. En un intento de solventar esta problemática, en Japón, llevan años realizando diferentes estudios para el desarrollo de una metodología sencilla para la selección de árboles tolerantes (Matsunaga et al., 2011; Matsunaga and Togashi, 2009). 
En ellos, la enfermedad del marchitamiento del pino se estudia a través de la determinación de la migración de nematodos en segmentos de ramas. Estos estudios sirvieron de base para los ensayos realizados en esta tesis, dado que en este país es donde tienen el mayor conocimiento de todos los factores implicados: patogenicidad de distintas cepas de B. xylophilus, época del año óptima para el desarrollo de este tipo de ensayos y existencia de una clasificación previa de clones en función de su tolerancia. Los ensayos de segmentos de rama de esta tesis fueron realizados en Japón (Kyushu Regional Breeding Office, Kumamoto) utilizando principalmente la especie $P$. thunbergii.

La metodología utilizada en estos ensayos no ha permitido encontrar una relación entre la migración de nematodos a través de los segmentos de ramas y la clase de tolerancia previamente conocida de los clones de $P$. thunbergii. Sin embargo, en los sí ha sido posible diferenciar entre un clon susceptible de $P$. thunbergii y otro tolerante. No obstante, es muy posible que este resultado se haya visto afectado por las condiciones climáticas previas a la recogida del material del experimento, ya que otros ensayos mostraron que el momento de realización de este tipo de experimentos es muy importante

La confirmación de esta relación en nuevos estudios permitiría una asignación rápida de nuevos clones a las distintas clases de tolerancia, lo cual sería muy conveniente a la hora de acelerar y abaratar los programas de mejora.

Concluyendo, consideramos que a falta de comprobar el efecto del clima en la enfermedad, el norte y noroeste de España tiene un alto riesgo de expansión de la enfermedad, por poseer zonas con grandes extensiones de $P$. pinaster, $P$. radiata y $P$. sylvestris, especies susceptibles o muy susceptibles. Esto podrá tener un grave impacto económico y ambiental en estas zonas. El resto del territorio español, centro (donde $P$. pinaster no esté presente) y este de España, así como las islas Canarias, es posible que no se vea afectado, o muy poco, por la enfermedad.

\section{Referencias bibliográficas}

Abelleira, A., Picoaga, A., Mansilla, J.P., Aguin, O., 2011. Detection of Bursaphelenchus xylophilus, causal agent of pine wilt disease on Pinus pinaster in Northwestern Spain. Plant Dis. 95, 776-776. https://doi.org/10.1094/PDIS-12-10-0902

Daub, M., 2008. Investigations on pathogenicity, invasion biology and population dynamics of the pine wood nematode Bursaphelenchus xylophilus (Steiner und Buhrer 1934) Nickle 1970 in European conifers. Tesis doctoral. Rheinischen Friedrich-Wilhelms-Universität Bonn.

EPPO, 2018. Isolated finding of Bursaphelenchus xylophilus in Castilla y Léon, (Spain). EPPO Reporting Service No. 07 - Pests \& Diseases, 2018/140. https://gd.eppo.int/repor ting/Rse-2018-07

Hakamata, T., Kato, K., Yamamoto, S., 2013. Correlation of seedling size and branch number with disease resistance of Pinus thunbergii seedlings to Bursaphelenchus xylophilus. For. Pathol. 43, 238-244. https://doi.org/10.1111/efp.12026 
Hopf-Biziks, A., Schröder, T., Schütz, S., 2016. The pine wood nematode, Bursaphelenchus xylophilus (Steiner \&amp; Buhrer) Nickle, and its pathogenicity to German Pinus sylvestris provenances. J. Plant Dis. and Protect. 123, 43-49. https://doi.org/10.1007/s413480 16-0005-4

Inácio, M., Nóbrega, F., Vieira, P., Bonifácio, L., Naves, P., Sousa, E., Mota, M., 2015. First detection of Bursaphelenchus xylophilus associated with Pinus nigra in Portugal and in Europe. For. Pathol. 45, 235-238. https://doi.org/10.1111/efp.12162

Kuroda, K., 2004. Inhibiting factors of symptom development in several Japanese red pine (Pinus densiflora) families selected as resistant to pine wilt. J. For. Res. 9, 217-224. https://doi.org/10.1007/s10310-004-0076-0

Linit, M.J., and Kinn, D.N., 1993. Influence of pinewood nematode (Nematoda: Aphelenchoididae) infection on the preformed defensive response of Loblolly Pine. Environ. Entomol. 22, 1285-1293. https://doi.org/10.1093/ee/22.6.1285

Mamiya, Y., 1983. Pathology of the pine wilt disease caused by Bursaphelenchus xylophilus. Annu. Rev. Phytopathol. 21, 201-220. https://doi.org/10.1146/annurev.py.21.090183. 001221

Matsunaga, K., Maezono, H., Tamaki, S., Togashi, K., 2011. Inhibition response of Pinus densiflora clones to Bursaphelenchus xylophilus systemic dispersal and their resistance to pine wilt disease. Nematology. 13, 653-659. https://doi.org/10.1163/138855410X535 732

Matsunaga, K., and Togashi, K., 2009. Seasonal change in susceptibility of Pinus densiflora to Bursaphelenchus xylophilus infection, determined from the number of nematodes passing through branch sections. Nematology. 11, 409-418. https://doi.org/10.1163/1568 54109X446980

Menéndez, M., Abelleira, A., Mansilla, P., Mosquera, P., Abelleira-Sanmartín, A., Alonso, M., Toval, G., Diaz, R., 2014. Susceptibility evaluation of Pinus pinaster families to Pinewood nematode (Bursaphelenchus xylophilus). 5th Internatinal Conference on Mediterranean Pines (medpine5). Solsona, Spain sept 22-26. Poster Session B Biotic Interactions.

Menéndez-Gutiérrez, M., Alonso, M., Toval, G., Díaz, R., 2017. Variation in pinewood nematode susceptibility among Pinus pinaster Ait. provenances from the Iberian Peninsula and France. Ann. For. Sci. 74:76. https://doi.org/10.1007/s13595-017-0677-3

Menéndez-Gutiérrez, M., Alonso, M., Jiménez, E., Toval, G., Mansilla, P., Abelleira, A., Abelleira-Sanmartín, A., Díaz, R., 2018a. Interspecific variation of constitutive chemical compounds in Pinus spp. xylem and susceptibility to pinewood nematode (Bursaphelenchus xylophilus). Eur. J. Plant Pathol. 150, 939-953. https://doi.org/10.1007/s10658017-1334-2

Menéndez-Gutiérrez, M., Alonso, M., Toval, G., Díaz, R., 2018b. Testing of selected Pinus pinaster half-sib families for tolerance to pinewood nematode (Bursaphelenchus xylophilus). Forestry. 91, 38-48. https://doi.org/10.1093/forestry/cpx030

Mori, Y., Miyahara, F., Tsutsumi, Y., Kondo, R., 2008. Relationship between resistance to pine wilt disease and the migration or proliferation of pine wood nematodes. Eur. J. Plant Pathol. 122, 529-538. https://doi.org/10.1007/s10658-008-9321-2

Naves, P., de Sousa, E., Quartau, J.A., 2006. Reproductive traits of Monochamus galloprovincialis (Coleoptera: Cerambycidae) under laboratory conditions. Bull. Entomol. Res. 96, 289-94. http://www.ncbi.nlm.nih.gov/pubmed/16768817. https://doi.org/10.1079/ BER2006422 
Nunes da Silva, M., Solla, A., Sampedro, L., Zas, R., Vasconcelos, M.W., 2015. Susceptibility to the pinewood nematode (PWN) of four pine species involved in potential range expansion across Europe. Tree Physiol. 35, 987-999. https://doi.org/10.1093/treephys/ tpv046

Panesar, T., and Sutherland, J., 1989. Pathogenicity of canadian isolates of the Bursaphelenchus xylophilus (pinewood nematode) to provenances of Pinus sylvestris and Pinus contorta as grown in Finland: A greenhouse study. Scand. J. Forest Res. 4, 549-557. https:// doi.org/10.1080/02827588909382587

Phillips, M.A., and Croteau, R.B., 1999. Resin-based defenses in conifers. Trends Plant Sci. 4, 184-190. https://doi.org/10.1016/S1360-1385(99)01401-6

Phrame, 2007. Development of improved pest risk analysis techniques for quarantine pests, using pinewood nematode, Bursaphelenchus xylophilus, in Portugal as a model system. https://www.forestry.gov.uk/pdf/PHRAMEJuly07.pdf/\$FILE/PHRAMEJuly07.pdf Accessed 10 June 2017

Pimentel, C.S., Firmino, P.N., Calvão, T., Ayres, M.P., Miranda, I., Pereira, H., 2016. Pinewood nematode population growth in relation to pine phloem chemical composition. Plant Pathol. 66, 856-864. https://doi.org/10.1111/ppa.12638

Pimentel, C.S., Gonçalves, E.V., Firmino, P.N., Calvão, T., Fonseca, L., Abrantes, I., Correia, O., Máguas, C., 2017. Differences in constitutive and inducible defences in pine species determining susceptibility to pinewood nematode. Plant Pathol. 66, 131-139. https:// doi.org/10.1111/ppa.12548

Son, J.A., and Moon, Y., 2013. Migrations and multiplications of Bursaphelenchus xylophilus and B. mucronatus in Pinus thumbergii in relation to their pathogenicity. Plant Pathol J. 29, 116-122. https://doi.org/10.5423/PPJ.OA.06.2012.0091

Toda, T., and Fujimoto, Y., 1987. Resistance to the pine wood nematodes in Pinus densiflora (Kirishima-matsu). Jap For. Soc. 98, 261-262.

Vivas, M., Zas, R., Solla, A., 2012. Screening of maritime pine (Pinus pinaster) for resistance to Fusarium circinatum, the causal agent of pitch canker disease. Forestry. 85, 185-192. https://doi.org/10.1093/forestry/cpr055

Woo, K.S., Lee, D.H., Koo, Y.B., Yeo, J.K., 2008. Inoculation of seven pine species or hybrid seedlings with Korean isolates of pinewood nematode under greenhouse conditions. Ann. For. Sci. 65, 811-811. https://doi.org/10.1051/forest:2008072

Xunta de Galicia. RESOLUCIÓN de 6 de junio de 2016, de la Dirección General de Ganadería, Agricultura e Industrias Agroalimentarias, por la que se declara en el territorio de la Comunidad Autónoma de Galicia la presencia de un nuevo brote del organismo de cuarentena Bursaphelenchus xylophilus (Steiner et Buhrer) Nickle et al. (nematodo del pino) y se ordena comenzar las medidas para su erradicación. Diario Oficial de Galicia, 21 de Junio de 2016, núm. 117, pp. 25643.

Yamanobe, T., 2009. Relationships between morphological traits and resistance to pine wood nematode in two Japanese pines. Eur. J. Plant Pathol. 124, 543-552. https://doi.org/10. 1007/s10658-009-9438-y

Zamora, P., Rodríguez, V., Renedo, F., Sanz, A.V., Domínguez, J.C., Pérez-Escolar, G., Miranda, J., Álvarez, A., González-Casas, A., Mayor, E., Dueñas, M., Miravalles, A., Navas, A., Robertson, A., Martín, A.B., 2015. First Report of Bursaphelenchus xylophilus causing pine wilt disease on Pinus radiata in Spain. Plant Dis. 99, 1449-1449. https:// doi.org/10.1094/PDIS-03-15-0252-PDN 
Zas, R., Moreira, X., Ramos, M., Lima, M.R.M., Nunes da Silva, M., Solla, A., Vasconcelos, M.W., Sampedro, L., 2015. Intraspecific variation of anatomical and chemical defensive traits in Maritime pine (Pinus pinaster) as factors in susceptibility to the pinewood nematode (Bursaphelenchus xylophilus). Trees. 29, 663-673. https://doi.org/10.1007/ s00468-014-1143-6

Zas, R., Sampedro, L., Prada, E., Fernández-López, J., 2005. Genetic variation of Pinus pinaster Ait. seedlings in susceptibility to the pine weevil Hylobius abietis L. Ann. For. Sci. 62, 681-688. https://doi.org/10.1051/forest:2005064 


\section{Datos de la tesis}

Premio Accésit de la SECF 2017 en el apartado de tesis doctoral.

Directora: Raquel Díaz Vázquez.

Centro: Centro de Investigación Forestal de Lourizán.

Fecha de defensa: Septiembre de 2017

\section{Publicaciones derivadas de la tesis}

Menéndez-Gutiérrez M., Alonso M., Jiménez E., et al. (2018a) Interspecific variation of constitutive chemical compounds in Pinus spp. xylem and susceptibility to pinewood nematode (Bursaphelenchus xylophilus). Eur J Plant Pathol 150:939-953. doi: 10.1007/s10658-017-1334-2

Menéndez-Gutiérrez M., Alonso M., Toval G., Díaz R. (2018b) Testing of selected Pinus pinaster half-sib families for tolerance to pinewood nematode (Bursaphelenchus xylophilus). For An Int J For Res 91:38-48. doi: 10.1093/forestry/cpx030

Menéndez-Gutiérrez M., Alonso M., Toval G., Díaz R. (2017a) Variation in pinewood nematode susceptibility among Pinus pinaster Ait. provenances from the Iberian Peninsula and France. Ann For Sci 74:76. doi: 10.1007/s13595-017-0677-3

Menéndez-Gutiérrez M., Matsunaga K., Togashi K. (2017b) Relationship between pine wilt-tolerance rankings of Pinus thunbergii trees and the number of Bursaphelenchus xylophilus passing through branch sections. Nematology 19:1083-1093. doi: 10.1163/15685411-00003108 